\title{
BCAS2 is essential for Drosophila viability and functions in pre-mRNA splicing
}

\author{
PO-HAN CHEN, ${ }^{1}$ CHIA-I LEE, ${ }^{1}$ YU-TZU WENG, ${ }^{1}$ WOAN-YUH TARN,${ }^{2}$ YEOU-PING TSAO, ${ }^{3}$ PING-CHANG KUO, ${ }^{1}$ \\ PANG-HUNG HSU, ${ }^{4,5}$ CHU-WEI HUANG, ${ }^{1}$ CHIUN-SHENG HUANG, ${ }^{6}$ HSIU-HSIANG LEE, ${ }^{7}$ JUNE-TAI WU, ${ }^{7,8}$ \\ and SHOW-LI CHEN ${ }^{1,9}$ \\ ${ }^{1}$ Graduate Institute of Microbiology, College of Medicine, National Taiwan University, Taipei 100, Taiwan \\ ${ }^{2}$ Institute of Biomedical Sciences, Academia Sinica, Taipei 115, Taiwan \\ ${ }^{3}$ Department of Ophthalmology, Mackay Memorial Hospital, Taipei 104, Taiwan \\ ${ }^{4}$ Department of Life Science, College of Life Sciences, National Taiwan Ocean University, Keelung 202, Taiwan \\ ${ }^{5}$ Institute of Bioscience and Biotechnology, College of Life Sciences, National Taiwan Ocean University, Keelung 202, Taiwan \\ ${ }^{6}$ Department of Surgery, College of Medicine, National Taiwan University and Hospital, Taipei 100, Taiwan \\ Institute of Molecular Medicine, College of Medicine, National Taiwan University, Taipei 100, Taiwan \\ ${ }^{8}$ Department of Medical Research, National Taiwan University Hospital, Taipei 100, Taiwan
}

\begin{abstract}
Here, we show that dBCAS2 (CG4980, human Breast Carcinoma Amplified Sequence 2 ortholog) is essential for the viability of Drosophila melanogaster. We find that ubiquitous or tissue-specific depletion of dBCAS2 leads to larval lethality, wing deformities, impaired splicing, and apoptosis. More importantly, overexpression of hBCAS2 rescues these defects. Furthermore, the C-terminal coiled-coil domain of hBCAS2 binds directly to CDC5L and recruits hPrp19/PLRG1 to form a core complex for splicing in mammalian cells and can partially restore wing damage induced by knocking down dBCAS2 in flies. In summary, Drosophila and human BCAS2 share a similar function in RNA splicing, which affects cell viability.
\end{abstract}

Keywords: BCAS2; Drosophila; viability; splicing; Prp19 complex

\section{INTRODUCTION}

BCAS2 is a $26-\mathrm{kDa}$ nuclear protein that contains two coiledcoil motifs and is expressed ubiquitously in normal human tissue (Kuo et al. 2009). Previously, we found that BCAS2 interacts directly with the tumor suppressor p53 and functions as a negative regulator (Kuo et al. 2009). Knocking down BCAS2 in wild-type p53 cancer cells, such as MCF7, LNCaP, and A549, induces programmed cell death. However, depletion of BCAS2 in p53-null cells (H1299) and p53 mutant (C33A) cells results in growth arrest at G2/M, suggesting that BCAS2 plays an essential role in the control of cell growth, in addition to the regulation of p53 (Kuo et al. 2009).

BCAS2 also was predicted to be a component of the spliceosome (Neubauer et al. 1998). The subspliceosomal complex, containing Prp19 (PSO4), Cef1 (CDC5L), Prp46 (PLRG1), and Snt309 (BCAS2), plays a critical role in yeast and human cells (Supplemental Fig. S1; Ajuh et al. 2000; Ohi and Gould 2002; Ohi et al. 2002, 2005; Grote et al. 2010). The yeast Prp19p-associated complex is required for

\footnotetext{
${ }^{9}$ Corresponding author

E-mail showlic@ntu.edu.tw

Article published online ahead of print. Article and publication date are at http://www.rnajournal.org/cgi/doi/10.1261/rna.034835.112.
}

stable association of the U5 and U6 snRNP with the spliceosome, after release of U4 snRNP (Chan et al. 2003; Chan and Cheng 2005). Disruption of the yeast BCAS2 ortholog (Cwf7 and Snt309) using genetic approaches results in the accumulation of pre-mRNA (Chen et al. 1998, 1999; Ohi and Gould 2002; Ohi et al. 2002). In addition, the Modifier of snc1 4 (MOS4) of Arabidopsis thaliana (the hBCAS2 ortholog in plants) has been shown to affect the splicing pattern of the resistance gene, sncl and rps4 in plants (Xu et al. 2012). Human Prp19, CDC5L, and PLRG1 are known to be required for splicing (Ajuh et al. 2000, 2001; Grillari et al. 2005), but whether BCAS2 is essential for pre-mRNA processing remains unclear.

In Drosophila melanogaster, CG4980 encodes the hBCAS2 ortholog, which is predicted to be a component of the dPrp19 complex (Herold et al. 2009). Therefore, we used the fly as a model system in this study to examine the role of BCAS2 in cell viability, as well as the structure-function relationship in pre-mRNA splicing. Our results show that dBCAS2 and hBCAS2 share a similar function in premRNA splicing. Depletion of dBCAS2 in the whole body leads to larval lethality, and knockdown of dBCAS2 using a tissuespecific promoter results in deformity phenotypes on notum and wings and in increased apoptosis in wing discs, which can be rescued by hBCAS2 with the restored RNA splicing 
function. Therefore, the abnormality caused by depletion of dBCAS2 is, at least in part, a consequence of impaired splicing.

\section{RESULTS}

\section{BCAS2 is essential for Drosophila viability}

A previous report indicated that depletion of hBCAS2 in wild-type p53 cells induces apoptosis, whereas in p53 mutant or null cells, it results in G2 growth arrest (Kuo et al. 2009). Therefore, in addition to the regulation of p53, we hypothesized that BCAS2 is also involved in the basic mechanisms that maintain regular cell growth. We used D. melanogaster as an animal model to investigate the function of BCAS2 in vivo. Drosophila BCAS2 encodes 278 amino acids $(\sim 31$ $\mathrm{kDa}$ ), and BLAST analysis demonstrated that the human and Drosophila BCAS2 protein orthologs are 59\% identical over the entire sequence (Supplemental Fig. S2). We then used transgenic RNAi flies to examine the role of dBCAS2 using the UAS-GAL4 system. We first used an Act5C-GAL4 driver to express UAS-dBCAS2 $2^{d s R N A}$ ubiquitously and determined whether dBCAS2 is essential for fly survival. As shown in Table 1, the ratio of offspring with either genotype Act5CGAL4/T(2;3)SM6-TM6B or UAS-dBCAS2 ${ }^{\text {dsRNA } / T(2 ; 3) S M 6-~}$ TM6B compared with Act5C-GAL4/UAS-dBCAS2 ${ }^{d s R N A}$ was expected to be 2:1. The majority of third instar larvae Act5C-GAL4/UAS-dBCAS2 ${ }^{d s R N A}$ died before the wandering stage, and $<10 \%$ of the expected number survived to the pupae stage at $\mathrm{P} 1-\mathrm{P} 2$. In the end, no viable Act5C-GAL4/ $U A S-d B C A S 2^{d s R N A}$ adult flies were observed, indicating that dBCAS2 is essential for fly viability and that complete knockdown of dBCAS2 is lethal. Because human and Drosophila BCAS2 are very similar (Supplemental Fig. S2), we next generated an hBCAS2 transgenic line to investigate whether hBCAS2 can rescue this lethality. We used $U A S-d B C A S 2^{d s R N A}, U A S-h B C A S 2 / T(2 ; 3) S M 6-T M 6 B$ crossed to Act5C-GAL4/T(2;3)SM6-TM6B and predicted that 310

\begin{tabular}{|c|c|c|}
\hline \multicolumn{3}{|c|}{$\begin{array}{l}\text { TABLE 1. The Act5C-GAL4 driven UAS-dBCAS2 } 2^{\text {dsRNA }} \text { in } \\
\text { Drosophila causes lethality }\end{array}$} \\
\hline Progeny & $\begin{array}{c}\text { Act5C-GAL4/T(2;3) } \\
\text { SM6-TM6B or UAS- } \\
\text { dBCAS2 } 2^{d s R N A} / T(2 ; 3) \\
\text { SM6-TM6B }\end{array}$ & $\begin{array}{c}\text { Act5C-GAL4/ } \\
\text { UAS- } \\
\text { dBCAS2 } \\
\text { dsRNA }\end{array}$ \\
\hline d & 2 & 1 \\
\hline Results (no.) & 560 & 0 \\
\hline \multicolumn{3}{|c|}{ 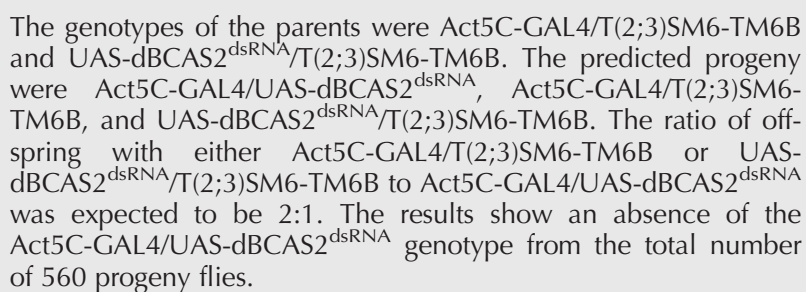 } \\
\hline
\end{tabular}

TABLE 2. hBCAS2 partially rescues lethality caused by the Act5CGAL4-driven $d B C A S 2^{\text {dsRNA }}$ in Drosophila

\begin{tabular}{|c|c|c|}
\hline Progeny & $\begin{array}{c}\text { Act5C-GAL4/T( }(2 ; 3) \\
\text { SM6-TM6B or } \\
\text { UAS-dBCAS2 } 2^{d S R N A}, \\
\text { UAS-hBCAS2/T(2;3) } \\
\text { SM6-TM6B }\end{array}$ & $\begin{array}{l}\text { Act5C-GAL4/ } \\
\text { UAS-dBCAS2 } 2^{d s R A} \\
\text { UAS-hBCAS2 }\end{array}$ \\
\hline Predicted ratio & 2 & 1 \\
\hline Results (no.) & 620 & 91 \\
\hline \multicolumn{3}{|c|}{$\begin{array}{l}\text { UAS-dBCAS2 }{ }^{\text {dsRNA }} \text {, UAS-hBCAS2/T(2;3)SM6-TM6B was crossec } \\
\text { with Act5C-GAL4/T(2;3)SM6-TM6B, and } 91 \text { Act5C-GAL4/UAS } \\
\text { dBCAS2 } 2 \text { dsRNA, UAS-hBCAS2 flies survived and showed no obvious } \\
\text { defects (data not shown). }\end{array}$} \\
\hline
\end{tabular}

(620/2) flies would express UAS-dBCAS2 $2^{d s R N A}, U A S-$ $h B C A S 2$. As shown in Table 2, we found that $29.4 \%(n=$ 91, 91/310) of the adult progeny Act5C-GAL4/UAS$d B C A S 2^{d s R N A}, U A S-h B C A S 2$ were able to survive with no obvious defective phenotype. Expression of $d B C A S 2$ and $h B C A S 2$ was confirmed in surviving progeny by RT-PCR (Supplemental Fig. S3). These results suggest that hBCAS2 could compensate for the loss of dBCAS2 function and thus partially rescue the lethality of dBCAS2-depleted flies.

In order to find visible phenotypes induced by knocking down dBCAS2, we chose a tissue-specific ms1096-GAL4 driver that is expressed in the pouch region of wing discs (Milan et al. 1998) to knock down dBCAS2. All of the progeny carrying UAS- $d B C A S 2^{d s R N A}$ exhibited growth defects at the notum and wings, such as an irregular scutellum margin (Fig. 1A, panel c) and twisted and shrunken wings (Fig. 1A, panel d). The apterous-GAL4 (ap-GAL4) driver that is expressed in the dorsal compartment of third instar larvae wing discs (Diaz-Benjumea and Cohen 1993) was selected to confirm UAS-dBCAS2 $2^{d s R N A}$-induced wing deformation. All the flies carrying UAS-dBCAS2 $2^{d s R N A}$ (Supplemental Fig. $\mathrm{S} 4 \mathrm{~B}, \mathrm{H}, \mathrm{N}$ ) showed defects such as discoloration of the notum; irregular scutellum margin; abnormal number and length of bristles, which appeared similar to Stubble (no Stubble mutant background) (Supplemental Fig. $\mathrm{S} 4 \mathrm{H}$ ); and twisted and shrunken wings (Supplemental Fig. S4N). However, flies expressing $h B C A S 2$ and $d B C A S 2^{d s R N A}$ under the control of ms1096-GAL4 had restored phenotypes with full recovery of the scutellum margin (Fig. 1A, panel g) and wing shape, compared with wild-type flies (Fig. 1A, panel a,b) and flies expressing hBCAS2 (Fig. 1A, panel e,f). But the wing veins in flies expressing $h B C A S 2$ and $d B C A S 2^{d s R N A}$ were only partially recovered, the anterior crossvein (ACV) between longitudinal veins L3 and L4 appearing to be absent (Fig. 1A, panel $\mathrm{h}$, arrow) and the posterior crossvein (PCV) between L4 and L5 looking truncated (Fig. 1A, panel h, arrowhead; Blair 2007). But when UAS-dBCAS2 $2^{d s R N A}, U A S-h B C A S 2$ was expressed using the ap-GAL4 driver (Supplemental Fig. S4D, E), we only observed partial rescue (Supplemental Fig. S4D, $\mathrm{E}$, arrow mark) of the wing shape. The different rescue effects 


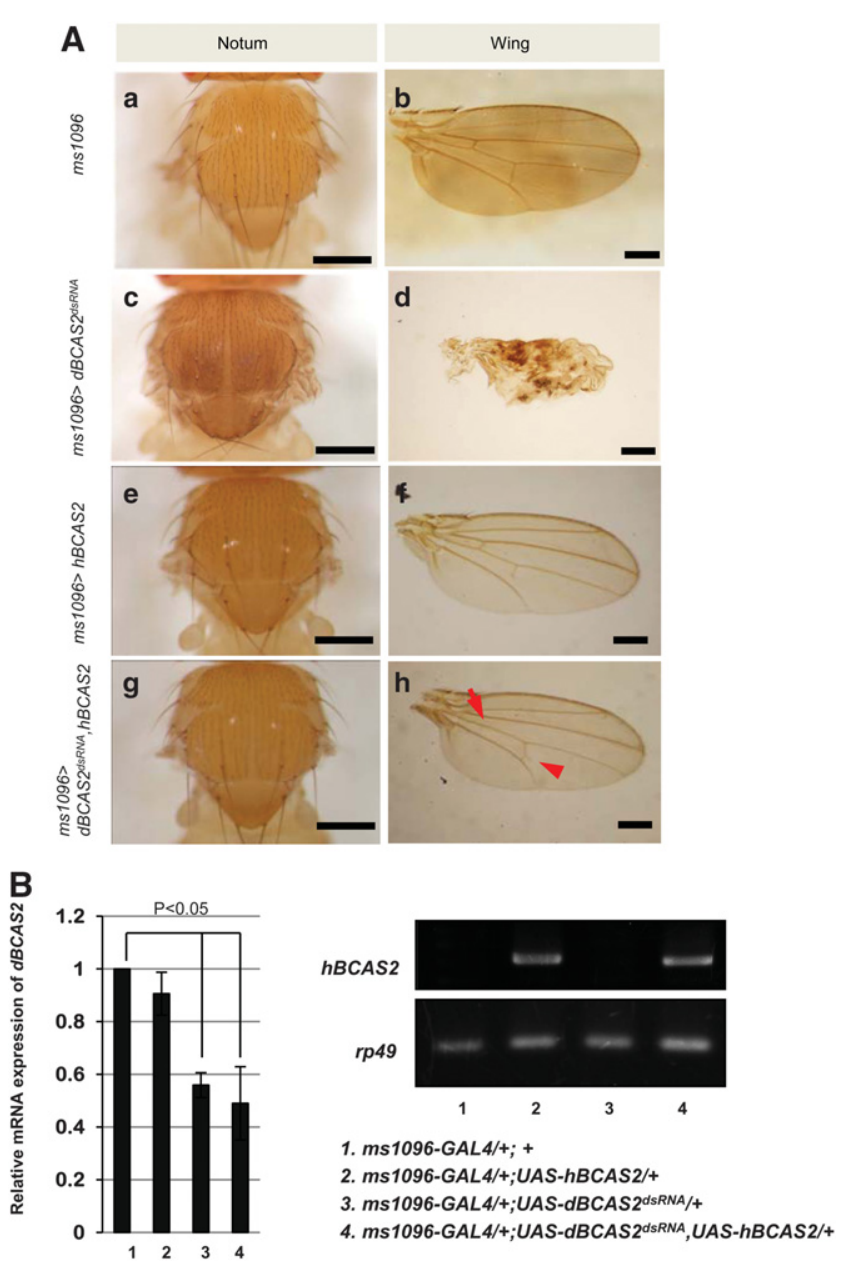

FIGURE 1. BCAS2 is essential for normal wing development. (A) $\mathrm{hBCAS} 2$ rescues growth defects in notum and wings induced by knocking down dBCAS2. Parental genotype (from top to bottom): ms1096-GAL4 crossed with either (1) $w^{1118}$, (2) UAS-dBCAS2 $2^{d s R N A} / T(2 ; 3) S M 6-$ TM6B, (3) UAS- $h B C A S 2$, or (4) UAS-dBCAS2 ${ }^{d s R N A}, U A S-h B C A S 2 / T$ (2;3)SM6-TM6B. Scale bar, $0.5 \mathrm{~mm}$. The red arrow indicates the anterior crossvein (ACV) between longitudinal veins L3 and L4, and the arrowhead indicates the posterior crossvein (PCV) between L4 and L5. (B) Expression of $d B C A S 2$ and $h B C A S 2$ described above. RNA was isolated from the wing discs of the third instar larvae, and mRNA of $d B C A S 2$ was measured by quantitative RT-PCR (left panel). (Upper right panel) The mRNA expression of $h B C A S 2$ was analyzed by RT-PCR. The internal control, rp49. (Lower right) Genotypes of each fly.

between these drivers (ms1096-GAL4, ap-GAL4, and Act5CGAL4) could be accounted for by the strength of the tissuespecific promoter during wing development.

Because UAS- $d B C A S 2^{d s R N A}$ and UAS- $h B C A S 2$ were driven by one set of GAL4, it is possible that the activity of the GAL4 activator was diluted and that the rescue effects resulted from decreased expression of small interfering RNAs. To exclude this possibility, we generated Drosophila ap-GAL4, UAS- $m C D 8-G F P / U A S-d B C A S 2^{d s R N A}$, which contained two UAS targeting sites, to drive UAS-mCD8-GFP and UAS$d B C A S 2^{d s R N A}$ simultaneously. The fly ap-GAL4,UAS- $m C D 8$ GFP/UAS-dBCAS2 $2^{d s R N A}$ (Supplemental Fig. S4F,L,R) showed similar defective phenotypes to those with UAS-dBCAS2 ${ }^{d s R N A}$ alone (Supplemental Fig. S4B,H,N) indicating that the rescue effect is not due to titration of the RNAi by $h B C A S 2$. In addition, the efficiency of dBCAS2 knockdown in $U A S-d B C A S 2^{d s R N A}$ (Fig. 1B, left panel, lane 3) was nearly the same as UAS-dBCAS2 ${ }^{d s R N A}, U A S-h B C A S 2$ (Fig. 1B, left panel, lane 4), as measured by quantitative RT-PCR. Likewise, hBCAS2 expression in UAS-dBCAS2 $2^{d s R N A}$ and $U A S-d B C A S 2^{d s R N A}, U A S-h B C A S 2$ was the same as analyzed by RT-PCR (Fig. 1B, right panel, lanes 2,4 ). The titration effects of GAL4 can be ruled out by using one or two UAS constructs. The dBCAS2 RNAi line \#26676 from VDRC has a potential off target hit against CG18375 (dASPP), which is highly expressed in wing discs, and mutants in dASPP demonstrate a wing overgrowth phenotype (Langton et al. 2007). To rule out this off-target effect, we measured the expression of two dASPP encoded transcripts (RA and RB) in dBCAS2knockdown flies using quantitative RT-PCR. As shown in Supplemental Figure S5, there was no significant change from the wild type, suggesting that the wing deformation caused by dBCAS2 RNAi line \#26676 is not an off-target effect on the expression of dASPP. Taken together, these findings indicate that human and Drosophila BCAS2 share a similar function and that human BCAS2 can rescue the defects caused by depleting Drosophila BCAS2.

\section{dBCAS2 is essential for RNA splicing}

Because dBCAS2 is predicted to be a member of the subspliceosomal complex, we determined next whether the defective growth of dBCAS2-knockdown flies is a consequence of impaired pre-mRNA splicing. To test this hypothesis, RNA extracted from escaper Act5C-GAL4/UAS-dBCAS2 $2^{d s R N A}$ third instar larvae was analyzed by quantitative RT-PCR. We chose the transcripts of $\gamma$-tubulin and hpo, as reported (Andersen and Tapon 2008), to test whether dBCAS2-knockdown larvae exhibited abnormal pre-mRNA splicing in vivo. We found that the mRNA of $\gamma$-tubulin and hpo decreased by $30 \%$ to $50 \%$, compared with the wild type, and the premRNA of $\gamma$-tubulin and hpo accumulated by 2.7 -fold and fourfold, respectively, in dBCAS2-knockdown larvae (Fig. 2A, black box). This suggests that lethality (Table 1) and wing deformation (Fig. 1A) induced by depletion of dBCAS2 may correlate with impaired splicing. We also investigated whether the rescue effect by hBCAS2 is related to repair splicing efficiency. Therefore, Act5C-GAL4/UAS$d B C A S 2^{d s R N A}, U A S-h B C A S 2$ third instar larvae were subjected to RNA splicing analysis. As shown in Figure 2A (gray box), both the pre-mRNA and mRNA of $\gamma$-tubulin and hpo recovered to wild-type levels (white box). This verifies our hypothesis that human and fly BCAS2 share a similar function in RNA processing, which is essential for cell viability. Consistent results of decreased $\gamma$-tubulin, hpo, and stg mRNA and accumulated $\gamma$-tubulin and hpo pre-mRNA were obtained from Drosophila S2 cells by knocking down 


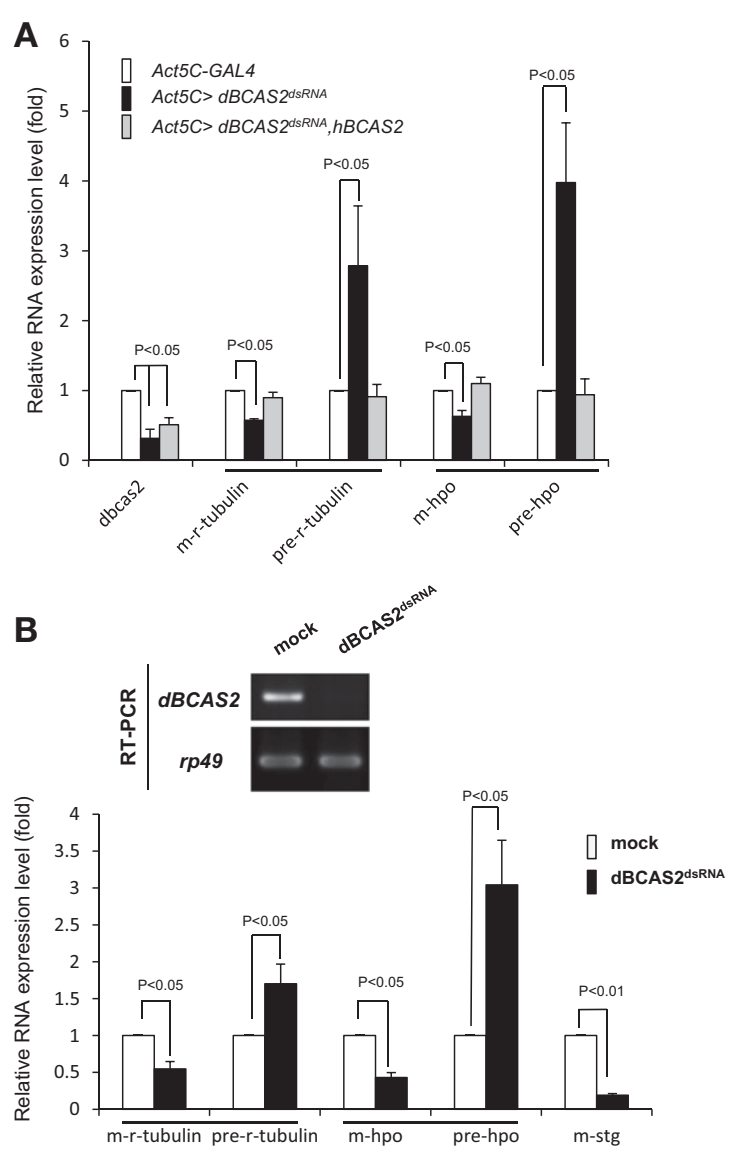

FIGURE 2. hBCAS2 plays a similar role as $\mathrm{dBCAS2}$, which is necessary for pre-mRNA splicing. (A) dBCAS2-knockdown larvae displays impaired pre-mRNA splicing, and hBCAS2 rescues splicing defects. Third instar larvae RNAs were extracted from Act5C-GAL4/UASdBCAS2 $2^{d s R N A}$ (black box), Act5C-GAL4/UAS-dBCAS2 ${ }^{d s R N A}, U A S-$ hBCAS2 (gray box), and Act5C-GAL4/+ (white box). $\gamma$-tubulin and hpo pre-mRNA and mRNA were analyzed by quantitative RT-PCR. (B) Depletion of dBCAS2 showed impaired pre-mRNA processing in S2 cells. (Upper panel) Knocking down dBCAS2 by dsRNA in Drosophila S2 cells. (Lower panel) $\gamma$-tubulin, hpo, and stg mRNA and $\gamma$-tubulin and hpo pre-mRNA were analyzed by quantitative RT-PCR. Data are shown as means and SD relative to wild-type controls from three independent experiments. The $P$-values estimated by the Student's $t$-test were statistically significant.

dBCAS2 using dsRNA (Fig. 2B, lower panel). However, similar to previous reports, the stg pre-mRNA level was not detectable by quantitative RT-PCR; this was suggested to be attributable to low levels or instability of the pre-mRNA (Andersen and Tapon 2008). The decreased expression of $d B C A S 2$ by RT-PCR was shown in the upper panel. Our data confirm that dBCAS2 plays a role in RNA splicing as a component of the subspliceosome (Herold et al. 2009).

\section{C-terminal hBCAS2 associates with hPrp19 complex and is necessary for RNA splicing}

To determine whether human BCAS2 is a bona fide component of the hPrp19 complex, we used GST-hBCAS2 pull down MCF-7 nuclear extracts and analyzed putative hBCAS2-interacting proteins by mass spectrometry. In addition to the hPrp19/CDC5L complex, we also found numerous RNA processing-related proteins, as shown in Table 3. We then performed immunoprecipitation analysis. As shown in Supplemental Figure S6, both the PLRG1 and hBCAS2 antibodies were able to pull down endogenous CDC5L, hPrp19, and PLRG1 in HEK (human embryonic kidney

TABLE 3. The potential hBCAS2-interacting proteins involved in RNA splicing

\begin{tabular}{|c|c|c|c|}
\hline Protein name & $\begin{array}{c}\text { Accession } \\
\text { no. } \\
\text { (Swissprot) }\end{array}$ & $\begin{array}{l}\text { No. of } \\
\text { peptides }^{\mathrm{a}}\end{array}$ & $\begin{array}{c}\text { Sequence } \\
\text { coverage (\%) }\end{array}$ \\
\hline \multicolumn{4}{|c|}{ hPrpl 9/CDC5L complex } \\
\hline CDC5L & Q99459 & 45 & 38 \\
\hline hPrp19 & Q9UMS4 & 8 & 14.3 \\
\hline AD-002 & Q9P013 & 2 & 4.3 \\
\hline \multicolumn{4}{|c|}{ SR and SR-related proteins } \\
\hline ASF/SF2 & Q07955 & 12 & 20.6 \\
\hline SRp55 (SFRS6) & Q13247 & 5 & 10.1 \\
\hline P54 (SFRS11) & Q6PJY9 & 3 & 1.7 \\
\hline U2AF1 & Q01081 & 5 & 16.7 \\
\hline 9G8 (SFRS7) & Q16629 & 15 & 26.1 \\
\hline CDC2L5 & Q9BVE2 & 5 & 8.4 \\
\hline SRp20 (SFRS3) & P84103 & 16 & 31.1 \\
\hline \multicolumn{4}{|l|}{ hnRNPs } \\
\hline hnRNP R & 043390 & 25 & 18.8 \\
\hline hnRNP U & Q00839 & 54 & 38.3 \\
\hline hnRNP F & P52597 & 3 & 8.7 \\
\hline hnRNP H1 & P31943 & 14 & 21.4 \\
\hline hnRNP H3 & P31942 & 4 & 9 \\
\hline hnRNP A3 & P51991 & 6 & 10.8 \\
\hline hnRNP A2/B & P22626 & 5 & 12.6 \\
\hline hnRNP core A & P09651 & 19 & 31.2 \\
\hline \multicolumn{4}{|l|}{ snRNP-associated } \\
\hline U5 snRNP 100 & Q9BUQ8 & 4 & 2.7 \\
\hline $\mathrm{kD}$ protein & & & \\
\hline U4/U6 snRNP & 043395 & 7 & 4.6 \\
\hline $\begin{array}{l}\text { 90kD protein } \\
\text { (Prp3) }\end{array}$ & & & \\
\hline SF3B14 & Q9Y3B4 & 12 & 44 \\
\hline \multicolumn{4}{|l|}{ RNA-binding protein } \\
\hline $\begin{array}{l}\text { RNA binding } \\
\text { motif protein } 25\end{array}$ & P49756 & 34 & 21.2 \\
\hline $\begin{array}{l}\text { RNA binding } \\
\text { motif protein 12B }\end{array}$ & Q8IXT5 & 7 & 4.6 \\
\hline $\begin{array}{l}\text { RNA binding } \\
\text { motif protein } 14\end{array}$ & Q96PK6 & 47 & 30.2 \\
\hline $\begin{array}{l}\text { RNA binding } \\
\text { motif protein } 39\end{array}$ & Q14498 & 16 & 17.7 \\
\hline $\begin{array}{l}\text { RNA binding } \\
\text { motif protein } 8 \mathrm{~A}\end{array}$ & Q9Y5S9 & 4 & 14.4 \\
\hline \multicolumn{4}{|c|}{ DEAD-box RNA helicase } \\
\hline DDX 39 & B1Q2N1 & 20 & 34.4 \\
\hline DDX17 & Q92841 & 29 & 26.9 \\
\hline
\end{tabular}

Mass spectrometry analysis of GST-hBCAS2 pull down proteins obtained from MCF-7 nuclear extracts shows several putative hBCAS2-interacting proteins involved in RNA processing.

${ }^{a}$ Number of peptides identified for each protein. 
epithelial cells) 293 cells (Supplemental Fig. S6, lanes 3,4), confirming that hPrp19, CDC5L, PLRG1, and hBCAS2 form a stable complex in mammalian cells. Because human BCAS2 contains two coiled-coil motifs, which can mediate protein-protein or protein-DNA interactions (Mason and Arndt 2004), in the C-terminal region (Fig. 3A), we used GST-hBCAS2, GST- $\triangle$ CC (deletion of two coiled-coil domains), GST-C, His-tagged hPrp19, and CDC5L proteins, generated from Escherichia coli, for in vitro protein-protein interactions to investigate in detail their association within the hPrp19 complex. The results showed that His-hPrp19 could bind either full-length hBCAS2 or $\triangle \mathrm{CC}$ protein (Fig. $3 \mathrm{~B}$, lanes 3,4 ), but not the $\mathrm{C}$-domain protein (Fig. 3B, lane
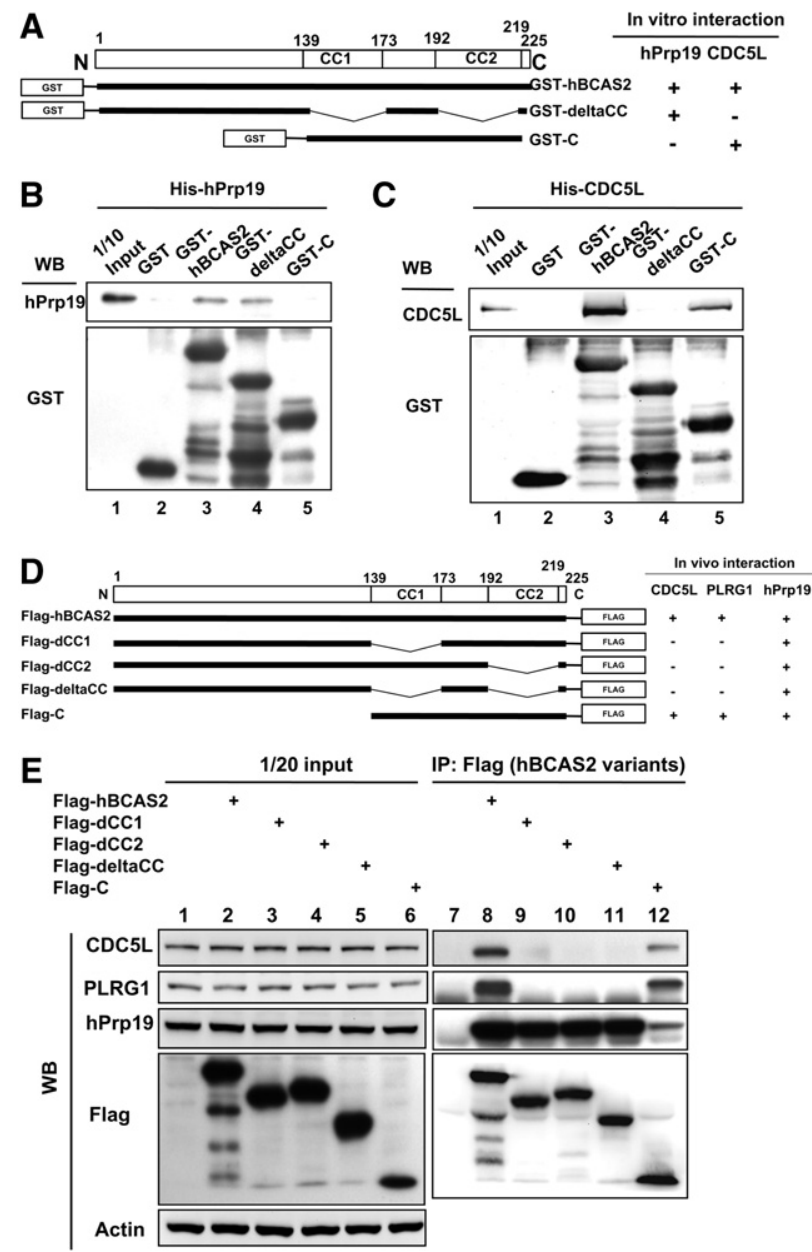

FIGURE 3. The C-terminal coiled-coil region of hBCAS2 associates with the hPrp19 complex. (A) Schematic representation of GST-tagged hBCAS2, $\triangle \mathrm{CC}$, and C constructs and summarized data. (B) Full-length and $\triangle \mathrm{CC}$ of hBCAS2 interacts directly with hPrp19. (C) Full-length and C-terminal hBCAS2 interacts directly with CDC5L. (D) Schematic representation of Flag-tagged hBCAS2, dCC1, dCC2, $\triangle \mathrm{CC}$, and C constructs. (Right) Summarized data of in vivo interactions from $E$. $(E)$ Mapping interaction domain between hBCAS2 and CDC5L, PLRG1, hPrp19 in vivo. 1/20 input was analyzed by Western blot as shown in the left panel (lanes 1-6). Proteins precipitated with Flag-tagged hBCAS2 variants were revealed by blotting using indicated antibodies (right panel, lanes 7-12).
5). On the other hand, His-CDC5L interacted directly with full-length hBCAS2 and the C-domain protein (Fig. 3C, lanes $3,5)$. The in vitro binding data are summarized in the right panel of Figure 3A. We conducted further in vivo binding experiments with members of the endogenous hPrp19 complex by transiently expressing Flag-tagged hBCAS2, dCC1, dCC2 (dCC1 and dCC2 plasmid constructs refer to deletion of coiled-coil domain 1 and 2, respectively), $\triangle \mathrm{CC}$, and Cdomain (Fig. 3D) constructs in HEK 293 cells. The results showed that the full-length hBCAS2 and C-domain protein associated with endogenous CDC5L, hPrp19, and PLRG1 (Fig. 3E, lanes 8,12). However, the coiled-coil deletion mutants Flag-dCC1, Flag-dCC2, and Flag- $\Delta$ CC only interacted with hPrp19 and not with CDC5L or PLRG1 (lanes 9-11). These in vivo results (summarized in Fig. 3D, right panel) were consistent with in vitro binding assays (Fig. 3A-C). Taken together, the hBCAS2 $\mathrm{N}$ terminus interacts only with hPrp19, whereas its C terminus (containing the coiled-coil domain) is necessary for interaction with all other major components of the hPrp19 complex.

To decipher the structure-function relationship of hBCAS2, the pSV40-CAT (In1) reporter (Fig. 4A, upper panel; Lin et al. 2004) was then used to investigate its function in constitutive splicing. The results showed that overexpressing hBCAS2 increased the pre-mRNA splicing efficiency of the reporter by approximately 1.45 -fold, and the $\mathrm{C}$ terminus enhanced this by 1.34 -fold, whereas the $\triangle \mathrm{CC}$ protein inhibited splicing to 0.69 -fold (Fig. 4A, lower panel). The corresponding hBCAS2 protein expression is shown in Figure 4B. In addition, we then used the adenovirus E1A as a reporter (Fig. $4 \mathrm{C}$, upper panel) to assay alternative splicing regulation of hBCAS2. Selection of the $5^{\prime}$ splice sites of E1A produces various amounts of $13 \mathrm{~s}, 12 \mathrm{~s}$, and $9 \mathrm{~s}$ mRNAs that are modulated by a variety of splicing regulatory factors (Lin et al. 2004). The results showed that hBCAS2 and its $\mathrm{C}$ terminus increased the ratio of $9 \mathrm{~s}$ mRNA, but decreased that of $12 \mathrm{~s}$ mRNA, from the E1A reporter (Fig. 4C,D). In general, when a splicing factor activates a distal $5^{\prime}$ splice site (i.e., the 9 s site), the level of 9s mRNA is increased with a concomitant reduction of both 12s and 13s mRNAs (Lai et al. 2003; Lin et al. 2004). However, hBCAS2 appeared to have no effect on the 13s mRNA, so that it only reduced $12 \mathrm{~s}$ and promoted $9 \mathrm{~s}$ expression, although the mechanism underlying this is not yet clear. Southern blotting also was performed to confirm the RTPCR products shown in Figure 4A and C (Supplemental Fig. S7A,B). To confirm the effect of hBCAS2 in alternative splicing endogenously, we also chose alternative transcripts associated with cell cycle control, because of the depletion of hBCAS2 causing G2 growth arrest (Kuo et al. 2009). The human Cdc25 phosphatase isoforms Cdc25B and Cdc25C are critical during the transition from G2 to $M$ phase (Boutros et al. 2007). Because our data in Figure 2B (lower panel) also showed that the mRNA level of stg (String, Drosophila Cdc25 ortholog) was dramatically decreased in dBCAS2-knockdown S2 cells, we determined by RT-PCR 
A
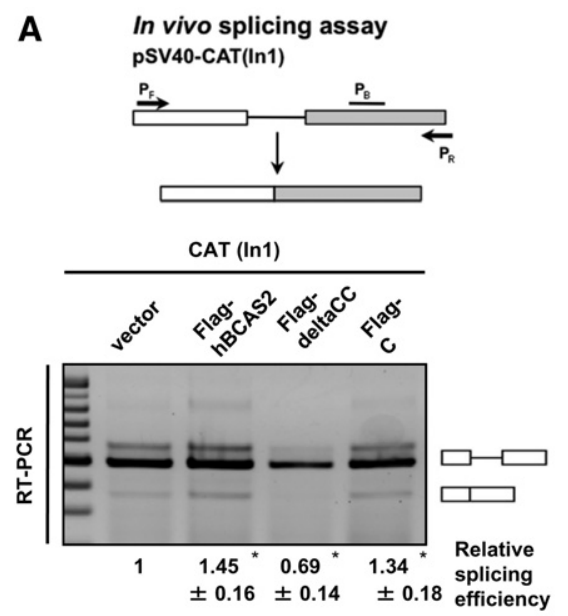

B

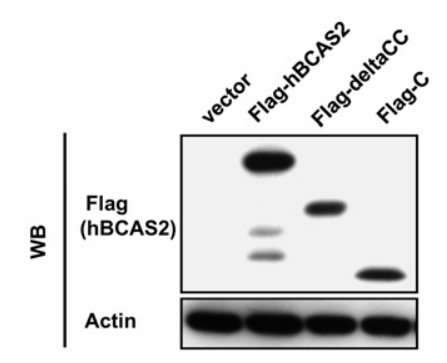

E

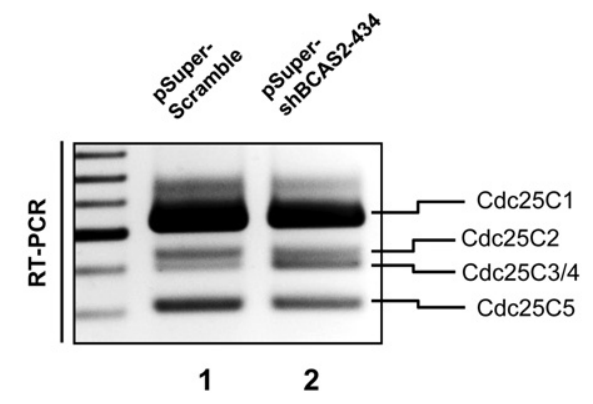

C
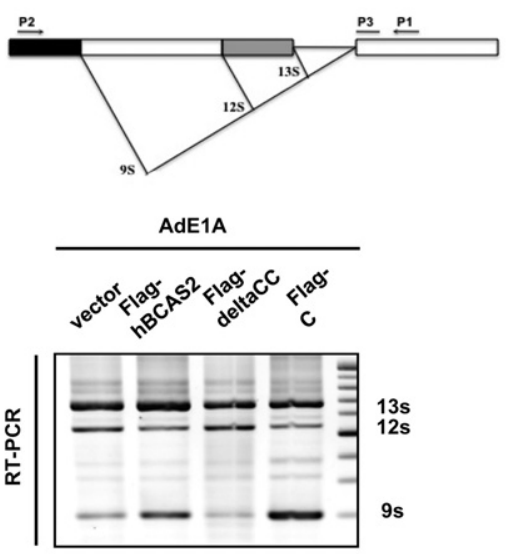

D
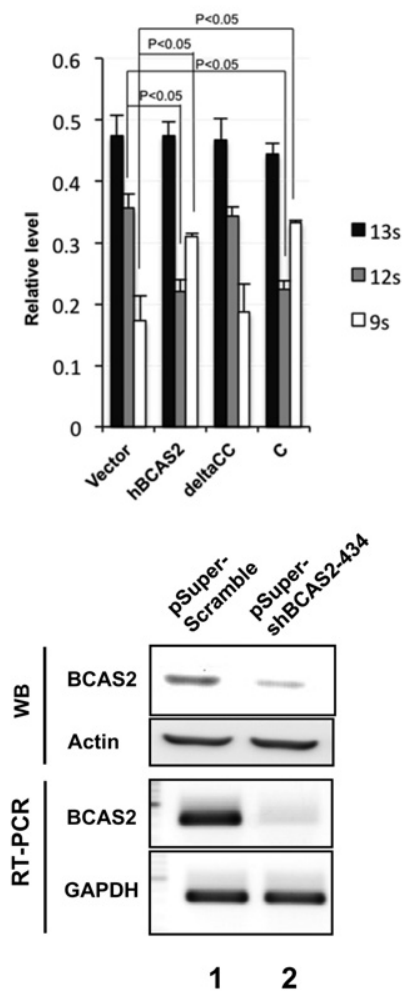

FIGURE 4. The $\mathrm{C}$ terminus is indispensable for the function of hBCAS2 in splicing. (A) C-terminal and full-length hBCAS2 enhances the CAT(In1) reporter pre-mRNA splicing. (Upper panel) Schematic representation of the CAT(In1) reporter. The splicing efficiency (lower panel) was calculated from the value of $\mathrm{mRNA} /(\mathrm{mRNA}+$ pre-mRNA), and the relative splicing efficiency was normalized to the vector control (set as 1 ). Data are shown as means and SD from three independent experiments. $\left({ }^{*} P<0.05\right)(B)$ Western blot analysis with anti-Flag antibody to determine ectopically expressed protein with the reporter in HEK 293 cells. (C) C-terminal and full-length hBCAS2 modulates $5^{\prime}$ splice site selection of the AdE1A reporter. Schematic representation of the AdE1A reporter is shown in the upper panel. (Lower panel) AdE1A reporter was cotransfected with either control vector or Flag-hBCAS2 variants into HEK 293 cells and analyzed by RT-PCR to determine $13 \mathrm{~s}, 12 \mathrm{~s}$, and $9 \mathrm{~s}$ RNAs. $(D)$ Quantitative results of alternative splicing. $C$ showed one represented experiment. The data of $D$ were collected from three independent experiments and shown as means and SD. $P$ values were estimated by the Student's $t$-tests and were statistically significant. $(E)$ Depletion of hBCAS2 influences the alternative splicing patterns of Cdc25C variants. The Cdc25C alternative splicing variants were examined by RT-PCR in BCAS2-depleted MCF7 cells. (Left) Knocking down BCAS2 (lane 2) alters the ratio of Cdc25C3/4 to Cdc25C1 variant compared with scramble control (lane 1). (Right) Protein and mRNA expression of BCAS2 and internal control are revealed by Western blot and RT-PCR.

whether hBCAS2 affected the splicing variants of Cdc25C (Pacheco et al. 2006; Albert et al. 2011). Our data revealed that knocking down hBCAS2 changed the ratio of $\mathrm{Cdc} 25 \mathrm{C} 3 / 4$ to $\mathrm{C} 1$ variants, compared with the scramble control, in MCF7 cells (Fig. 4E, left panel, lane 2; Supplemental Fig. S7C). The right panel shows that hBCAS2 was efficiently depleted by transient transfection of pSuper-shBCAS2-434 (Fig. 4E, right panel, lane 2) at both the mRNA and protein levels. This verifies the role of hBCAS2 in alternative splicing. In summary, the hBCAS2 C terminus containing the coiled-coil motifs is critical for RNA splicing and association with the hPrp19 complex.

\section{The C terminus of hBCAS2 partially restores defective phenotypes in ABCAS2-depleted flies}

Because the $\mathrm{C}$ terminus is indispensable for the function of hBCAS2 in RNA splicing (Fig. 5A), we asked the question whether the C-fragment of hBCAS2 rescues defects in dBCAS2-depleted flies in the same way as full-length hBCAS2. We generated an hBCAS2-C transgenic fly that showed no obvious phenotypic change compared with the wild type (data not shown). When UAS-hBCAS2$C$ and UAS-dBCAS2 ${ }^{d s R N A}$ were expressed with an ms1096-GAL4 driver, the wing deformation was partially rescued, in that the shrunken and wrinkled wings became partially unfolded and the wing veins could be clearly observed, compared with the dBCAS2-knockdown fly (Fig. 5B, panels b,d); however, the notum still exhibited a similar phenotype (Fig. 5B, cf. panels a and c). Expression of hBCAS2-C mRNA in the wing discs of ms1096-GAL4-driven $U A S-d B C A S 2^{d s R N A}$ and UAS- $h B C A S 2-C$ flies was confirmed by RT-PCR (Fig. 5C, lane 2). This indicates that the hBCAS2-C fragment can partially rescue the wing deformation of dBCAS2-depleted flies. To confirm that the partial rescue effect of the hBCAS2-C fragment is correlated with the splicing function, we examined next the splicing efficiency in third instar larvae expressing UAS- 
A

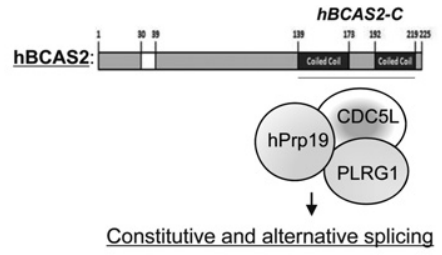

B
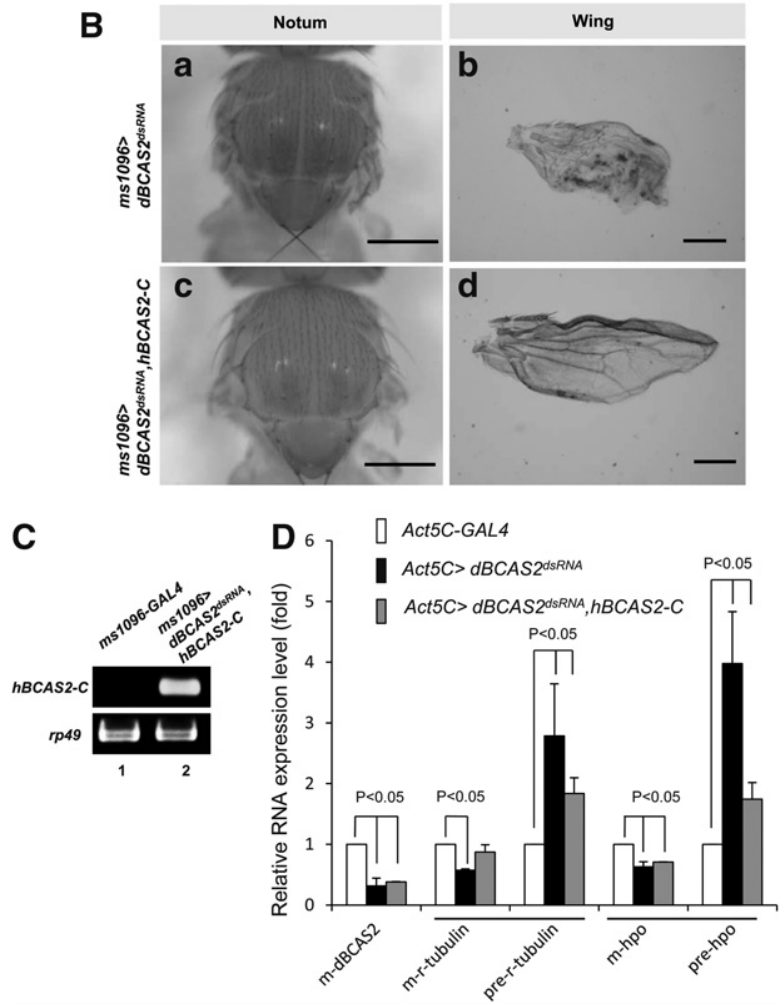

FIGURE 5. C-terminal hBCAS2 partially rescues dBCAS2-knockdown induced wing and splicing defects. $(A) \mathrm{C}$-terminal hBCAS2 plays a core of hPrp19 complex for RNA splicing. (B) Ectopic expression of hBCAS2-C partially rescues $A B C A S 2^{d S R N A}$-induced wing deformation. $U A S-h B C A S 2-C$ and $U A S-d B C A S 2^{d s R N A}$ were expressed under the control of ms1096-GAL4. Scale bar, $0.5 \mathrm{~mm}$. (C) Expression of hBCAS2-C in ms1096-GAL4/+;UAS-dBCAS2 $2^{d s R N A} /+; U A S-h B C A S 2-C /+$. RNAs were extracted from wing discs of third instar larvae and subjected to RT-PCR to confirm the expression of hBCAS2-C (lane 2) compared with the wild-type control (lane 1). The internal control, $r p 49$. (D) Cterminal hBCAS2 partially rescues impaired splicing in dBCAS2-depleted third instar larvae. Act5C-GAL4/UAS-dBCAS2 ${ }^{\text {dsRNA }}$;UAS- $h B C A S 2-$ $C /+$ (gray box) third instar larvae RNA were extracted and analyzed by quantitative RT-PCR as described in the legend to Figure 2.

$d B C A S 2^{d s R N A}$ and UAS- $h B C A S 2-C$, driven by Act5C-GAL4. As shown in Figure 5D, C-terminal hBCAS2 (gray box) partially rescued the level of mRNA and pre-mRNA of $\gamma$-tubulin and $h p o$, compared with UAS-dBCAS2 $2^{d s R A}$ alone. On the other hand, we also examined the level of viability restored by the hBCAS2-C fragment in the Act5C-GAL4/ $d B C A S 2^{d s R N A}$. We screened 241 adult flies, and in contrast with the $29.4 \%$ restored survival rate of full-length hBCAS2, the progeny expressing $d B C A S 2^{d_{s} R N A}$ and $h B C A S 2-C$ had not survived (data not shown). This indicates that the $\mathrm{C}$-frag- ment appears less potent than the full-length protein in restoring fly viability. Taken together, the rescue effect with hBCAS2-C is weaker than with the full-length protein, yet hBCAS2-C appears as potent as the full-length protein in the mammalian cell splicing assays. The differences of BCAS2 function between fly and mammalian cells are interesting subject for further investigation.

\section{dBCAS2 knocked down cells have a higher degree of apoptosis}

Previous studies have shown that depletion of splicing factors such as dMAFP1 and dPrp38 affects Drosophila cell proliferation and induces apoptosis (Andersen and Tapon 2008). We have found that depletion of BCAS2 resulted in splicing defects and wing deformation. Other than the observation of the splicing defect, notum, and wing phenotypes, we examined further cell viability in the wing discs of third instar larvae when knocking down dBCAS2. The engrailed-GAL4 was used as a driver and is located the posterior part of wing discs where UAS-dBCAS2 ${ }^{d s R N A}$ (Fig. 6B), UAS-dBCAS2 ${ }^{d s R N A}, U A S-$ $h B C A S 2$ (Fig. 6C), and UAS-dBCAS2 ${ }^{d s R N A}, U A S-h B C A S 2-C$ (Fig. 6D) were expressed. As shown in Figure 6, knocking down dBCAS2 significantly increased the number of apoptotic cells, as revealed by staining cleaved caspase 3 (Fig. 6F, red spot), compared with the wild type (Fig. 6E). However, coexpressing hBCAS2 (Fig. 6G) or hBCAS2-C (Fig. 6H) could fully or partially reduce cleaved caspase 3-positive cells in dBCAS2-knockdown wing discs. Because human BCAS2 was reported to be a negative regulator of the tumor suppressor p53 in cancer cell lines (Kuo et al. 2009), it is possible that the apoptosis phenomenon in dBCAS2-knockdown wing discs results from direct effects of dBCAS2 on the Drosophila p53 (dmp53), rather than splicing defects. To rule out the effect of dmp53, we observed caspase 3 activity when knocking down dBCAS2 by ms1096-GAL4 in dmp53null mutant flies (Sogame et al. 2003). As shown in Supplemental Figure S8C, the cleaved caspase 3 staining in dBCAS2-depleted flies (Supplemental Fig. S8B) was still higher than the control (Supplemental Fig. S8A) in the dmp53-null mutant background. In summary, our results provide evidence that the function of BCAS2 in RNA splicing is essential for Drosophila cell viability.

\section{DISCUSSION}

\section{BCAS2 is essential for Drosophila viability and RNA splicing}

In this study, we showed that dBCAS2 is essential for Drosophila viability via shRNA-mediated depletion in the whole body (Table 1). In addition, conditional knocking down of dBCAS2 using ms1096-GAL4, ap-GAL4, or engrailed-GAL4 drivers results in damaged phenotypes and 


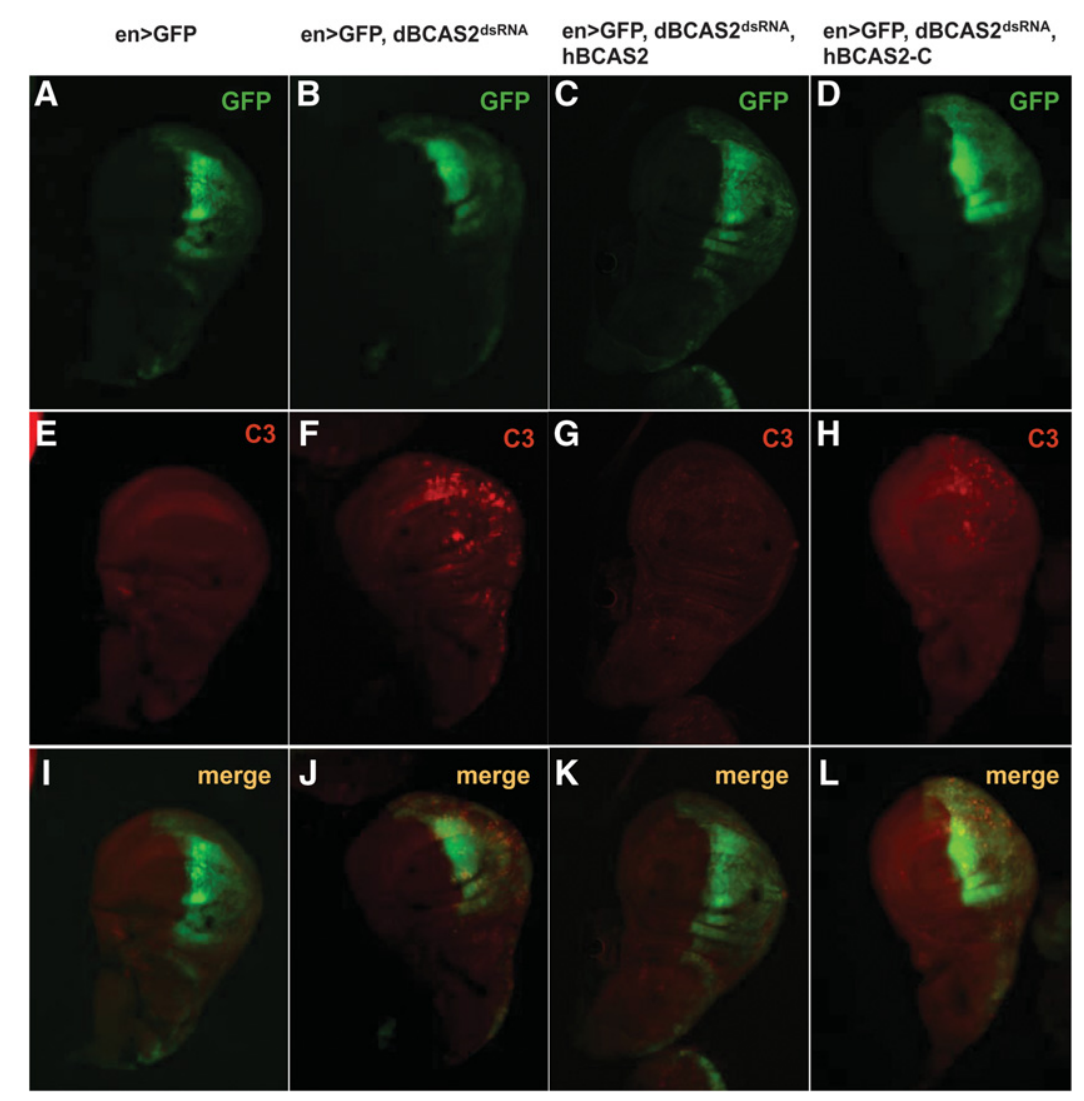

FIGURE 6. dBCAS2 knock down cells display higher level of apoptosis. Apoptotic cells were visualized by cleaved caspase 3 staining in dBCAS2-knockdown wing discs, and the expression of hBCAS2 or hBCAS2-C reduced cleaved caspase 3-positive staining. engrailed-GAL4 drives GFP (green, panels $A-D$ ) locating the posterior (to the right of discs) region of wing discs where $U A S$-dBCAS2 $2^{d s R N A}$ (panels $\left.B, F, J\right), U A S$-dBCAS2 ${ }^{d s R N A}, U A S$ - $h B C A S 2$ (panels $C, G, K$ ), and UAS$d B C A S 2^{d s R N A}, U A S-h B C A S 2-C$ (panels $D, H, L$ ) are expressed compared with the wild type (panels $A, E, I)$ from wandering third instar larvae. Anti-cleaved caspase 3 staining (red, panels $E-H$ ) revealed apoptotic cells in imaginal wing discs. Merged images (panels $I-L$ ). The genotypes of the flies are described in the Materials and Methods.

increased apoptotic cells in wings and imaginal discs, respectively (Figs. 1, 6; Supplemental Fig. S4; Table 1). These deformed phenotypes or lethality could be rescued by human BCAS2 (Figs. 1, 6; Supplemental Fig. S4; Table 2), indicating that hBCAS2 and dBCAS2 share similar functions that affect cell viability. Previously, we found that hBCAS2 is a negative regulator of p53 through direct interaction. Depletion of hBCAS2 increases p53 protein activity and induces p53-targeted gene expression, resulting in apoptosis. (Kuo et al. 2009). However, knocking down human BCAS2 in p53null and mutant cells induces G2/M arrest, suggesting that the function of BCAS2 in growth control may involve a mechanism other than the regulation of p53. BCAS2 (also named Spf27) was reported to be a component of the multi-protein spliceosome complex by mass spectrometry and EST-database searching, but its role in RNA splicing remains unclear (Neubauer et al. 1998). Here, we confirmed that human BCAS2 is an integral component of the hPrp19 subspliceosomal complex (Supplemental Fig. S6; Table 3), and we provide evidence that hBCAS2 participates in pre-mRNA splicing (Fig. 4). Furthermore, due to the conservation of human and fly BCAS2, we found depleting Drosophila BCAS2 in third instar larvae and in S2 cells caused decreased processing of mRNA and accumulation of pre-mRNA (Fig. 2), and overexpressing hBCAS2 in dBCAS2-depleted flies can restore the defects in RNA processing (Fig. 2), as well as the phenotypes in wings and lethality (Fig. 1; Table 2). Previous reports have illustrated that depletion of spliceosomal factors, such as ASF and dMFAP1, cause G2/M arrest (Bernstein and Coughlin 1998; Li et al. 2005; Andersen and Tapon 2008). Similarly, other members of the hPrp19 complex, hPrp19 and PLRG1, which are both required for RNA splicing, caused lethality at an early stage of embryo development when knocked out in mice (Fortschegger et al. 2007; Kleinridders et al. 2009). These reports support our data that the impairment of RNA splicing when BCAS2 is knocked down in p53null cells causes lethality or malformations in dBCAS2-depleted flies.

\section{Structure-function relationship of BCAS2}

As reported previously (Grote et al. 2010), the amino acid sequences of hBCAS2 and its counterpart Snt309

(Saccharomyces cerevisiae-simpler yeast), which lacks coiled-coil domains, share only $24 \%$ similarity. Although Snt309 is a component of the Prp19 complex, it does not interact directly with Cef1 (CDC5L ortholog) (Ohi et al. 2005). Therefore, we deduce from our data that the C-terminal coiled-coil domains of hBCAS2 contribute primarily to the interaction with CDC5L. Previous reports have shown that depletion of Snt309 protein by anti-Snt309 antibodies does not impair pre-mRNA splicing in vitro (Chen et al. 1998, 1999). Unlike Snt309, human BCAS2 enhances the efficiency of pre-mRNA splicing (Fig. 4). Thus, the coiled-coil domains of hBCAS2 are critical for its splicing function because of its direct interaction with CDC5L.

Moreover, the Cwf7 (BCAS2 counterpart) of the more complex yeast, Schizosaccharomyces pombe, contains sequences similar to the C-terminal domain of human BCAS2. Cwf7 can interact with the CDC5L ortholog and can rescue the growth defects of snt309-deficient cells (Ohi and Gould 2002; Ohi et al. 2002), suggesting that this BCAS2 ortholog 
has evolved to achieve functional complexity. This possibility is supported by the high similarity between Drosophila, plant (Palma et al. 2007), and human BCAS2, which all contain coiled-coil domains that can interact directly with CDC5L. In addition, the Drosophila Protein Interaction Mapping (DPiM) project has identified putative dBCAS2-interacting proteins, including CG6905 (CDC5L) and Tango4 (PLRG1), which are similar to GST-hBCAS2 pull down proteins (Table 3). Combined with our data (Figs. 3-5), these reports verify the structural and functional conservation between ABCAS2 and hBCAS2.

In this study, we identified a novel function for the constitutive splicing factor human BCAS2 in the $5^{\prime}$ splice site selection of an adenovirus E1A reporter (Fig. 4C). We also provide preliminary evidence that depletion of hBCAS2 affects the ratio of Cdc25C alternative transcripts (Fig. 4E), suggesting that BCAS2 has potential functions in the regulation of alternative splicing. Alternative splicing is an important posttranscriptional regulation that diversifies gene expression in higher eukaryotes. Two crucial factors control alternative splicing: the sequence and position of cis-acting elements and the local concentration and post-translational status of trans-acting factors. In general, trans-acting factors that regulate alternative splicing include SR (Ser-Arg) proteins and heterogeneous nuclear ribonucleoproteins (hnRNPs) particles (Chen and Manley 2009). Recent studies showed that hPrp19 interacts directly with Blom7 $\alpha$, a novel hnRNP $\mathrm{K}$ homology domain-containing protein that can modulate the alternative $5^{\prime}$ and $3^{\prime}$ splice site choices of reporter minigenes (Grillari et al. 2009). In addition, CDC5L/PLRG1 interacts with hnRNP $\mathrm{M}$ and affects the selection of alternative splice site (Lleres et al. 2010). It would be interesting to investigate whether BCAS2 regulates alternative splicing by affecting the interaction of POS4-Blom7 $\alpha$ or CDC5L/PLRG1hnRNPM. As BCAS2 only harbors two coiled-coil domains and does not belong to the families of SR proteins or hnRNPs, we still cannot exclude the possibility that the activity of the hBCAS2-C protein in alternative splicing and in restoration of the knockdown phenotype may involve its interaction with other splicing factors (as provided in our GST-hBCAS2 pull down list, Table 3), rather than known factors such the Prp19 complex. However, it may be possible that BCAS2 affects the overall kinetics of splicing as a result of the reduction or increase of a general splicing factor.

A previous report concluded that the hPrp19 core complex, including hBCAS2 (lack of $\mathrm{N}$-terminal six amino acids), the N-terminal domain of hPrp19, the C-terminal domain of CDC5L, and the N-terminal domain of PLRG1, forms a salt stable bounded mixture and resists a high concentration of the protease subtilisin (Grote et al. 2010). Consistently, our results demonstrate that the $\mathrm{C}$ terminus of hBCAS2 (containing the coiled-coil domains) has the capacity to interact directly with CDC5L, which in turn binds with hPrp19 and PLRG1 (Fig. 3) to carry out RNA splicing activities, comparable to full length hBCAS2 (Figs. 3, 4). This indicates that the capacity for core complex formation is responsible for regular splicing in mammalian cells. But in the Drosophila system, flies transgenic for full-length hBCAS2 (Fig. 1A, panel e) and hBCAS2-C (data not shown) show no effects in terms of changes in morphology, perhaps because of endogenous splicing complexes reaching saturation. However, the rescue effects were measured by crossing transgenic full-length or hBCAS2-C flies with dBCAS2-depleted flies, and the restoration of wing-deformation by hBCAS2-C (Fig. $5 \mathrm{~B}$, panel d) is weaker than that by the full-length hBCAS2 (Fig. 1A, panel $\mathrm{h})$. The rescue effect of hBCAS2-C is not as potent as fulllength hBCAS2, suggesting that there are other roles for BCAS2 in wing shape development, besides RNA splicing. Aside from RNA splicing, BCAS2 also has been reported to interact with estrogen receptor (ER) as a transcriptional cofactor (Qi et al. 2005) and with p53 as a negative regulator (Kuo et al. 2009). ER is known as a growth survivor and p53 as a tumor suppressor, both of these proteins are crucial for cell viability. The detailed roles of BCAS2 regulating the other genes involved in cell growth or development still are being investigated. Moreover, the N fragment of hBCAS2 interacts directly with hPrp19 and seems to have a dominantnegative role in RNA splicing (Fig. 4A,C) in mammalian cells. It would be interesting to investigate further whether the hBCAS2-N fragment binds with hPrp19, resulting in the dilution of the core complex components and impeding RNA splicing in the fly assay.

\section{MATERIALS AND METHODS}

\section{Fly strains}

Drosophila stocks were kept and crossed at $25^{\circ} \mathrm{C}$ and supplied with cornmeal medium. UAS- $d B C A S 2^{d s R N A}$ was purchased from Vienna Drosophila RNAi Center (stock no. 26676) and balanced over T(2;3) SM6-TM6B, a translocated chromosome 2-3 balancer. UAS$h B C A S 2$ and UAS-hBCAS2-C were constructed by the insertion of $h B C A S 2$ and $h B C A S 2-C$ (278 bp, amino acids 139-225) cDNA from HeLa cells into $p U A S T$ vectors and randomly inserted into the second and third chromosome of $w^{1118}$, respectively (kindly provided by the Taiwan Fly Core). UAS-dBCAS2 ${ }^{d s R N A}, U A S$ $h B C A S 2$ was established by the recombination of UAS$d B C A S 2^{d s R N A}$ with UAS-hBCAS2 and balanced over T(2;3)SM6TM6B. UAS-dBCAS2 ${ }^{d s R N A}$ and UAS-hBCAS2-C were balanced over T(2;3)SM6-TM6B on chromosome II and III, respectively, to generate UAS-dBCAS2 $2^{d s R N A} ; U A S-h B C A S 2-C / T(2 ; 3)$ SM6-TM6B; therefore, UAS-dBCAS2 ${ }^{d s R N A}$ and UAS-hBCAS2-C cosegregate during crosses. UAS-dBCAS2 $2^{d s R N A}, U A S-h B C A S 2$, or UAS-hBCAS2-C expressing flies could be distinguished by the selectable marker, Tubby. The $d m p 53^{-n s}$ strain was the kind gift of Dr. John M. Abrams (Sogame et al. 2003). ap-GAL4, ms1096-GAL4, Act5CGAL4, engrailed-GAL4, and $w^{1118}$ used in this study were obtained from Bloomington Drosophila stock center and maintained by the Fly Core Facility in the College of Medicine, National Taiwan University. Phenotypes of flies were examined $48 \mathrm{~h}$ post eclose by stereomicroscope (Leica) after being anesthetized by carbon dioxide. 
Wings isolated from adult flies were mounted with Hoyer's mounting medium before imaging. To validate the expression of UAShBCAS2 and UAS-ABCAS2 driven by ms1096-GAL4, total RNAs were extracted from wing discs of third instar larvae and analyzed by RT-PCR.

\section{Cell culture and plasmid constructs}

HEK 293 and MCF7 (breast cancer cells) were grown in Dulbecco's modified Eagle's medium and RPMI 1640 medium, respectively, supplied with $10 \%$ FBS, L-glutamine, and penicillin/streptomycin at $37^{\circ} \mathrm{C}, 5 \% \mathrm{CO}_{2}$. To knock down dBCAS2 in S2 cells, fulllength dBCAS2 cDNA from Drosophila S2 cells was used for dsRNA synthesis by in vitro transcription (Promega). dBCAS2 dsRNA was incubated with S2 cells for $5 \mathrm{~d}$ before RNA analysis according to the protocol reported previously (Rogers and Rogers 2008). GST-tagged and Flag-tagged hBCAS2 variants were constructed according to the method described previously (Kuo et al. 2009). Primers used in this study are listed in the Supplemental Information.

\section{Protein-protein interaction and antibody}

$6 \times$ His-CDC5L, hPrp19, and GST fusion protein (hBCAS2 variants) were prepared from E. coli BL21 (DE3) for in vitro interaction. GSTfusion protein conjugated beads were washed with sonication buffer (50 mM at pH $8.0 \mathrm{NaH}_{2} \mathrm{PO}_{4}, 300 \mathrm{mM} \mathrm{NaCl}, 20 \%$ glycerol, and DTT, PMSF) twice and once with co-IP buffer (20 mM Tris at pH 7.5, 150 $\mathrm{mM} \mathrm{NaCl}, 10 \%$ glycerol, $0.1 \% \mathrm{NP}-40,5 \mathrm{mM} \mathrm{MgCl}_{2}, 1 \mathrm{mM}$ EDTA, and $1 \mathrm{mM} \mathrm{DTT}$ ) and then incubated with eluted His-tagged proteins. After incubation, samples were washed and eluted for further Western blot analysis. HEK 293 cells transfected with Flag-hBCAS2 variants by calcium phosphate were subjected for in vivo immunoprecipitation (Kuo et al. 2009): anti-hBCAS2, hPrp19, PLRG1 (Bethyl Laboratories); anti-CDC5L (BD, ABcam); anti-GST, Flag M2, Actin (Sigma).

\section{In vivo splicing assays}

For detection of mRNA and pre-mRNA, RNA was extracted from three to five third instar larvae of each following genotypes: (1) Act5C-GAL4/+, (2) Act5C-GAL4/UAS-dBCAS2 $2^{d s R N A}$, (3) Act5CGAL4/UAS-dBCAS2 $2^{d s R N A}, U A S-h B C A S 2$, and (4) Act5C-GAL4/ $U A S-d B C A S 2^{d s R N A} ; U A S-h B C A S 2-C /+$. Two micrograms of RNA was treated with DNase (RQ1, Promega) before reverse transcription (SuperScript III, Invitrogen) and then analyzed by quantitative PCR (KAPA SYBR FAST qPCR, Kapa Biosystems) according to the manufacturer's instructions. Data were collected from technical triplication of each reaction of at least three independent samples. Primers for the detection of mRNA and pre-mRNA of $\gamma$-tubulin and $h p o$ were based on previous reports (Andersen and Tapon 2008). Details for in vivo constitutive and alternative splicing assays were described in previous reports (Lin et al. 2004). For alternative splicing of endogenous Cdc25C, MCF7 cells were transfected with either pSuper-scramble or pSuper-shBCAS2-434 by electroporation and incubated for 48-72 h before Western blot and RT-PCR analysis. Primers used were following previous reports (Pacheco et al. 2006; Albert et al. 2011).

\section{Immunohistochemistry}

Wing imaginal discs were dissected from wandering third instar larvae of each of the following genotypes: (1) engrailed-GAL4,UASGFP/+, (2) engrailed-GAL4,UAS-GFP/UAS-dBCAS2 ${ }^{d s R N A}$, (3) engrailed-GAL4,UAS-GFP/UAS-dBCAS2 ${ }^{d s R N A}, U A S-h B C A S 2$, and (4) engrailed-GAL4,UAS-GFP/UAS-dBCAS2 $2^{d s R N A}$;UAS-hBCAS2-C/+. The discs were then fixed for 20 min in PBS with $4 \%$ paraformaldehyde and then blocked in PBS with $0.3 \%$ Triton X and 5\% BSA (bovine serum albumin) for $30 \mathrm{~min}$ at room temperature and incubated with anti-cleaved caspase 3 antibody (1:600; Abcam) overnight at $4^{\circ} \mathrm{C}$. After washing with PBS-Triton $\mathrm{X}$ three times, they were incubated with Cy3-conjugated goat anti-rabbit IgG (1:1000, Jackson ImmunoResearch Lab) for $1 \mathrm{~h}$ at room temperature. The fluorescent image was acquired with a fluorescence microscope (Zeiss AXIO SCOPE A1).

\section{Mass spectrometry analysis of hBCAS2-associated protein}

Identification of putative hBCAS2-interacting proteins was performed first by in vitro recombinant GST-hBCAS2 pull down nuclear extracts from MCF7 breast cancer cells then eluted for SDS-PAGE separation and in-gel digestion. The digested peptides were then analyzed by high-resolution and high-mass accuracy nanoflow LCMS/MS (Liquid Chromatography-Tandem Mass Spectrometry, Thermo Fisher Scientific).

\section{SUPPLEMENTAL MATERIAL}

Supplemental material is available for this article.

\section{ACKNOWLEDGMENTS}

This work was supported by the National Science Council (NSC993112-B-002-037, NSC99-2628-B-002-023-MY3), National Taiwan University (98R0066-13; 99R311001, 98F008-201), and National Taiwan University Hospital (UN101-066). We thank Dr. ShuChun Teng for critical discussion and appreciate the technical support of the staff of the Sixth Core Lab and DNA Sequencing Facility, Department of Medical Research, NTUH. We thank Dr. John M. Abrams for his kind gifts. We also thank the Taiwan Fly Core Facility and Fly Core Facility in NTU for microinjection and maintaining fly strains. We thank Dr. Tim J. Harrison for editing our English.

Received June 10, 2012; accepted November 14, 2012.

\section{REFERENCES}

Ajuh P, Kuster B, Panov K, Zomerdijk JC, Mann M, Lamond AI. 2000. Functional analysis of the human CDC5L complex and identification of its components by mass spectrometry. EMBO J 19: 65696581.

Ajuh P, Sleeman J, Chusainow J, Lamond AI. 2001. A direct interaction between the carboxyl-terminal region of CDC5L and the WD40 domain of PLRG1 is essential for pre-mRNA splicing. J Biol Chem 276: 42370-42381. 
Albert H, Santos S, Battaglia E, Brito M, Monteiro C, Bagrel D. 2011. Differential expression of CDC25 phosphatases splice variants in human breast cancer cells. Clin Chem Lab Med 49: 1707-1714.

Andersen DS, Tapon N. 2008. Drosophila MFAP1 is required for pre-mRNA processing and G2/M progression. J Biol Chem 283: 31256-31267.

Bernstein HS, Coughlin SR. 1998. A mammalian homolog of fission yeast Cdc5 regulates G2 progression and mitotic entry. J Biol Chem 273: 4666-4671.

Blair SS. 2007. Wing vein patterning in Drosophila and the analysis of intercellular signaling. Annu Rev Cell Dev Biol 23: 293-319.

Boutros R, Lobjois V, Ducommun B. 2007. CDC25 phosphatases in cancer cells: Key players? Good targets? Nat Rev Cancer 7: 495507.

Chan SP, Cheng SC. 2005. The Prp19-associated complex is required for specifying interactions of U5 and U6 with pre-mRNA during spliceosome activation. J Biol Chem 280: 31190-31199.

Chan SP, Kao DI, Tsai WY, Cheng SC. 2003. The Prp19p-associated complex in spliceosome activation. Science 302: 279-282.

Chen M, Manley JL. 2009. Mechanisms of alternative splicing regulation: Insights from molecular and genomics approaches. Nat Rev Mol Cell Biol 10: 741-754.

Chen HR, Jan SP, Tsao TY, Sheu YJ, Banroques J, Cheng SC. 1998. Snt309p, a component of the Prp19p-associated complex that interacts with Prp19p and associates with the spliceosome simultaneously with or immediately after dissociation of $\mathrm{U} 4$ in the same manner as Prp19p. Mol Cell Biol 18: 2196-2204.

Chen HR, Tsao TY, Chen CH, Tsai WY, Her LS, Hsu MM, Cheng SC. 1999. Snt309p modulates interactions of Prp19p with its associated components to stabilize the Prp19p-associated complex essential for pre-mRNA splicing. Proc Natl Acad Sci 96: 5406-5411.

Diaz-Benjumea FJ, Cohen SM. 1993. Interaction between dorsal and ventral cells in the imaginal disc directs wing development in Drosophila. Cell 75: 741-752.

Fortschegger K, Wagner B, Voglauer R, Katinger H, Sibilia M, Grillari J. 2007. Early embryonic lethality of mice lacking the essential protein SNEV. Mol Cell Biol 27: 3123-3130.

Grillari J, Ajuh P, Stadler G, Loscher M, Voglauer R, Ernst W, Chusainow J, Eisenhaber F, Pokar M, Fortschegger K, et al. 2005. $\mathrm{SNEV}$ is an evolutionarily conserved splicing factor whose oligomerization is necessary for spliceosome assembly. Nucleic Acids Res 33: $6868-6883$.

Grillari J, Loscher M, Denegri M, Lee K, Fortschegger K, Eisenhaber F, Ajuh P, Lamond AI, Katinger H, Grillari-Voglauer R. 2009. Blom7a is a novel heterogeneous nuclear ribonucleoprotein $\mathrm{K}$ homology domain protein involved in pre-mRNA splicing that interacts with SNEV Prp19-Pso4. J Biol Chem 284: 29193-29204.

Grote M, Wolf E, Will CL, Lemm I, Agafonov DE, Schomburg A, Fischle W, Urlaub H, Luhrmann R. 2010. Molecular architecture of the human Prp19/CDC5L complex. Mol Cell Biol 30: 2105-2119.

Herold N, Will CL, Wolf E, Kastner B, Urlaub H, Luhrmann R. 2009. Conservation of the protein composition and electron microscopy structure of Drosophila melanogaster and human spliceosomal complexes. Mol Cell Biol 29: 281-301.

Kleinridders A, Pogoda HM, Irlenbusch S, Smyth N, Koncz C, Hammerschmidt M, Bruning JC. 2009. PLRG1 is an essential regulator of cell proliferation and apoptosis during vertebrate development and tissue homeostasis. Mol Cell Biol 29: 3173-3185.
Kuo PC, Tsao YP, Chang HW, Chen PH, Huang CW, Lin ST, Weng YT, Tsai TC, Shieh SY, Chen SL. 2009. Breast cancer amplified sequence 2 , a novel negative regulator of the p53 tumor suppressor. Cancer Res 69: 8877-8885.

Lai MC, Kuo HW, Chang WC, Tarn WY. 2003. A novel splicing regulator shares a nuclear import pathway with SR proteins. EMBO J 22: 1359-1369.

Langton PF, Colombani J, Aerne BL, Tapon N. 2007. Drosophila ASPP regulates C-terminal Src kinase activity. Dev Cell 13: 773-782.

Li X, Wang J, Manley JL. 2005. Loss of splicing factor ASF/SF2 induces G2 cell cycle arrest and apoptosis, but inhibits internucleosomal DNA fragmentation. Genes Dev 19: 2705-2714.

Lin KT, Lu RM, Tarn WY. 2004. The WW domain-containing proteins interact with the early spliceosome and participate in pre-mRNA splicing in vivo. Mol Cell Biol 24: 9176-9185.

Lleres D, Denegri M, Biggiogera M, Ajuh P, Lamond AI. 2010. Direct interaction between hnRNP-M and CDC5L/PLRG1 proteins affects alternative splice site choice. EMBO Rep 11: 445-451.

Mason JM, Arndt KM. 2004. Coiled coil domains: Stability, specificity, and biological implications. Chembiochem 5: 170-176.

Milan M, Diaz-Benjumea FJ, Cohen SM. 1998. Beadex encodes an LMO protein that regulates Apterous LIM-homeodomain activity in Drosophila wing development: A model for LMO oncogene function. Genes Dev 12: 2912-2920.

Neubauer G, King A, Rappsilber J, Calvio C, Watson M, Ajuh P, Sleeman J, Lamond A, Mann M. 1998. Mass spectrometry and EST-database searching allows characterization of the multi-protein spliceosome complex. Nat Genet 20: 46-50.

Ohi MD, Gould KL. 2002. Characterization of interactions among the Cef1p-Prp19p-associated splicing complex. RNA 8: 798-815.

Ohi MD, Link AJ, Ren L, Jennings JL, McDonald WH, Gould KL. 2002. Proteomics analysis reveals stable multiprotein complexes in both fission and budding yeasts containing Myb-related Cdc5p/Ceflp, novel pre-mRNA splicing factors, and snRNAs. Mol Cell Biol 22: 2011-2024.

Ohi MD, Vander Kooi CW, Rosenberg JA, Ren L, Hirsch JP, Chazin WJ, Walz T, Gould KL. 2005. Structural and functional analysis of essential pre-mRNA splicing factor Prp19p. Mol Cell Biol 25: 451-460.

Pacheco TR, Moita LF, Gomes AQ, Hacohen N, Carmo-Fonseca M. 2006. RNA interference knockdown of hU2AF35 impairs cell cycle progression and modulates alternative splicing of Cdc25 transcripts. Mol Biol Cell 17: 4187-4199.

Palma K, Zhao Q, Cheng YT, Bi D, Monaghan J, Cheng W, Zhang Y, Li X. 2007. Regulation of plant innate immunity by three proteins in a complex conserved across the plant and animal kingdoms. Genes Dev 21: 1484-1493.

Qi C, Zhu YT, Chang J, Yeldandi AV, Rao MS, Zhu YJ. 2005. Potentiation of estrogen receptor transcriptional activity by breast cancer amplified sequence 2. Biochem Biophys Res Commun 328: 393-398.

Rogers SL, Rogers GC. 2008. Culture of Drosophila S2 cells and their use for RNAi-mediated loss-of-function studies and immunofluorescence microscopy. Nat Protoc 3: 606-611.

Sogame N, Kim M, Abrams JM. 2003. Drosophila p53 preserves genomic stability by regulating cell death. Proc Natl Acad Sci 100: 4696-4701.

Xu F, Xu S, Wiermer M, Zhang Y, Li X. 2012. The cyclin L homolog MOS12 and the MOS4-associated complex are required for the proper splicing of plant resistance genes. Plant J 70: 916-928. 

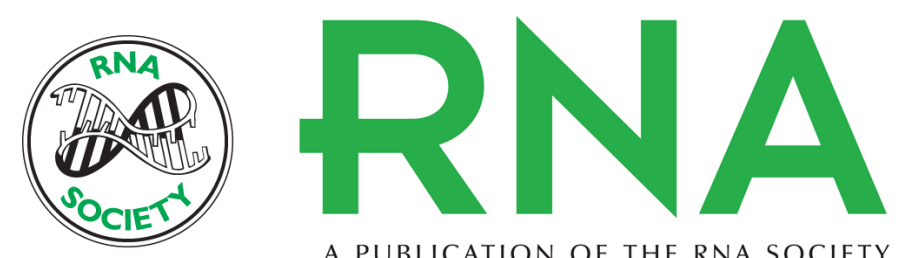

A PUBLICATION OF THE RNA SOCIETY

\section{BCAS2 is essential for Drosophila viability and functions in pre-mRNA splicing}

Po-Han Chen, Chia-I Lee, Yu-Tzu Weng, et al.

RNA 2013 19: 208-218 originally published online December 17, 2012

Access the most recent version at doi:10.1261/rna.034835.112

Supplemental
Material http://rnajournal.cshlp.org/content/suppl/2012/11/30/rna.034835.112.DC1

References This article cites 37 articles, 24 of which can be accessed free at:

http://rnajournal.cshlp.org/content/19/2/208.full.html\#ref-list-1

\section{License}

Email Alerting Receive free email alerts when new articles cite this article - sign up in the box at the Service top right corner of the article or click here.

To subscribe to $R N A$ go to:

http://rnajournal.cshlp.org/subscriptions 